\title{
ASSISTÊNCIA NUTRICIONAL AMBULATORIAL AO GRUPO MATERNO INFANTIL: RELATÓRIO DE ATIVIDADES
}

\author{
OUTPATIENT NUTRITIONAL ASSISTANCE TO MOTHERS AND \\ CHILDREN: ACTIVITIES REPORT
}

\section{Natalia Rodrigues Cardozo
Fernanda Weber Bordini ${ }^{2}$ Mariele dos Santos Rosa Xavier ${ }^{3}$ Inaê Dutra Valério ${ }^{4}$ Andriele Madruga Peres ${ }^{5}$ Thays Torres do Vale Oliveira ${ }^{6}$ Sandra da Costa Valle ${ }^{7}$ Juliana dos Santos Vaz ${ }^{8}$}

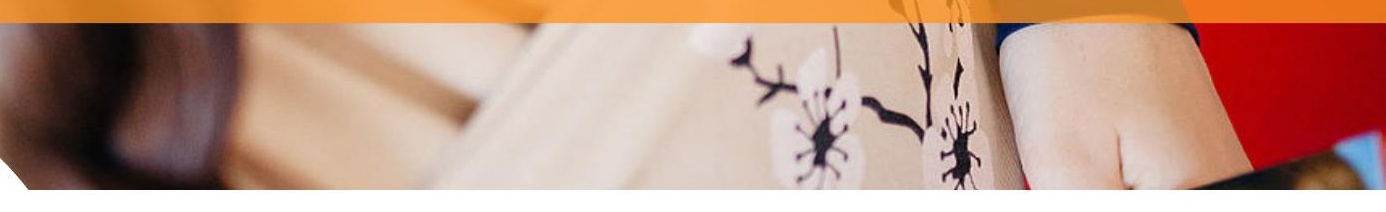

RESUMO

O presente relatório visa a descrever as ações desenvolvidas pelo projeto de extensão "Assistência nutricional ambulatorial a crianças" desenvolvido pela Faculdade de Nutrição junto ao ambulatório de Pediatria da Faculdade de Medicina (FAMED) da Universidade Federal de Pelotas (UFPel). O projeto tem como objetivo principal realizar atendimento nutricional a crianças e gestantes encaminhados pelos serviços de Pediatria e Ginecologia da FAMED e de unidades básicas de saúde da cidade de Pelotas e região. $O$ atendimento é realizado semanalmente, mediante agendamento. Além de atendimentos individualizados, realizam-se ações de atendimento em grupo na perspectiva da educação alimentar e nutricional e saúde bucal. No ano de $2016,65 \%$ das crianças foram encaminhadas com diagnóstico de sobrepeso ou obesidade, $6 \%$ desnutrição, $13 \%$ autismo - transtorno do déficit de atenção com hiperatividade ou neuropatias - $8 \%$ erros alimentar, entre outros. Na supervisão nutricional pré-natal, $46 \%$ das mulheres apresentavam obesidade anterior à gestação e foram encaminhadas para manejo do ganho de peso. Nas ações em saúde bucal, $56 \%$ das crianças atendidas foram encaminhadas para atendimento em consultório devido à cárie dentária. As ações desse projeto preenchem uma lacuna importante na assistência ambulatorial de crianças e gestantes de alto risco na comunidade de Pelotas e região.

Palavras chaves: Nutrição materno infantil. Atendimento multidisciplinar. Estado nutricional.

\footnotetext{
${ }^{1}$ Acadêmica do Curso de Nutrição da Universidade Federal de Pelotas (UFPel). Bolsista da Pró-reitoria de Extensão da UFPel no ano de 2016. Email: natalia.rodrigues.card@gmail.com

${ }^{2}$ Acadêmica do Curso de Nutrição da UFPel. Bolsista da Pró-reitoria de Extensão da UFPel no ano de 2016. Email: fernandawbordini@gmail.com

${ }^{3}$ Acadêmica do Curso de Nutrição da UFPel. Bolsista de Iniciação Científica pelo Programa Interinstitucional de Bolsas de Iniciação a Pesquisa da UFPel no ano de 2016. Email: marii_xavier@hotmail.com
} 


\section{ABSTRACT}

The extension project entitled "Nutritional outpatient care for children" is developed by the Faculty of Nutrition and conducted at the Pediatric outpatient clinic of the Faculty of Medicine, Federal University of Pelotas. The project aims to provide nutritional assistance for children and pregnant women referred by the Pediatrics and Gynecology Services and Primary Care Units of the city of Pelotas and region. The patient care is carried out weekly by appointment. Asides from individual nutritional assistance, the Project also develops group activities focused on nutritional and dietary education and oral health. In the year of $2016,65 \%$ of children were referred for diagnosis of overweight or obesity, $6 \%$ malnutrition, $13 \%$ autism, attention deficit hypertension disorder or neuropathies, $8 \%$ inadequacy of food habits, among others. In the prenatal nutritional supervision, $46 \%$ of women presented pre-gestational obesity and were referred for weight gain management. In oral health actions, $56 \%$ of the children assisted were referred for individual dental care due to caries. The activities conducted by this Project fill an important gap in the assistance of children and high-risk pregnant women living in the city of Pelotas and region.

Keywords: Maternal child nutrition. Multidisciplinary care. Nutritional status.

\section{INTRODUÇÃO}

Conhecer o estado nutricional e as condições de saúde do grupo materno infantil é de suma importância para os serviços de saúde, visto que as estatísticas de saúde deste grupo refletem o nível de desenvolvimento do país, como o crescimento econômico e as mudanças sociais, e estão correlacionadas à saúde geral da população (VICTORA et al., 2011).

No grupo materno infantil o estado nutricional e o ganho de peso gestacional merecem especial atenção. $O$ ganho de peso excessivo na gestação está associado a inúmeras complicações maternas, como desordens hipertensivas, diabetes gestacional, parto cirúrgico, além de conferir risco à saúde do bebê, como nascer grande para a idade gestacional

\footnotetext{
${ }^{4}$ Acadêmica do Curso de Nutrição da Universidade Federal de Pelotas. Bolsista de Ensino da Pró-reitoria de Graduação da UFPel no ano de 2016. Email: inadutra@hotmail.com

${ }^{5}$ Nutricionista residente do Programa de Residência Multiprofissional em Saúde - Atenção à Saúde da Criança do Hospital Escola da UFPel. Email: andrieleperes@gmail.com

${ }^{6}$ Cirurgiã-dentista residente do Programa de Residência Multiprofissional em Saúde - Atenção à Saúde da Criança do Hospital Escola da UFPel. Email: thaystorresdovale@hotmail.com.

${ }^{7}$ Docente da Faculdade de Nutrição, UFPel. Doutora em Ciências Biológica (Fisiologia) pela Universidade Federal do Rio Grande do Sul. Email: sandracostavalle@gmail.com

${ }^{8}$ Docente da Faculdade de Nutrição, UFPel. Doutora em Ciências Nutricionais pela Universidade Federal do Rio de Janeiro. Email: juliana.vaz@gmail.com
} 
e macrossômico. Por outro lado, o ganho de peso insuficiente é um fator de risco para a prematuridade e o baixo peso ao nascer (DREHMER; DUNCAN; KAC et al., 2013; KUBO; FERRARA; WINDHAM et al, 2014).

Tais ocorrências na gestação estão relacionados a qualidade e o número insuficiente de consultas pré-natais que para a maioria das gestantes brasileiras ainda está abaixo do preconizado (SILVEIRA; SANTOS; BARROS et al., 2008; CARVALHO; COELHO; SOARES et al., 2016).

Quanto ao estado nutricional infantil, no Brasil e em diversos países do mundo observa-se um crescimento da obesidade (OMS, 2016). Um estudo conduzido na cidade de Pelotas encontrou uma prevalência de sobrepeso de $20,6 \%$ e obesidade de $17,1 \%$ em crianças aos oito anos de idade (KAUFMANN; ALBERNAZ, 2013). Tais estimativas concordam com a prevalência de excesso de peso observada em outras cidades brasileiras (SILVEIRA; BARBOSA; VIEIRA; 2015; SALVADOR; KITOKO; GAMBARDELLA, 2014). Parte deste problema pode ser atribuída à educação nutricional insuficiente e o ambiente em que as crianças estão se desenvolvendo. A ausência de regulamentação na propaganda e venda de alimentos não saudáveis e bebidas industrializadas direcionados ao público infantil estão entre os fatores responsáveis pelo excesso de peso na população infantil (CABRAL; BRAGAGLIA; SEABRA, 2012).

Com o aumento da obesidade na população, cresce a demanda nos serviços de saúde, incluindo a procura por atendimento nos serviços de Nutrição do Sistema Único de Saúde (SUS) (DE SENA; PEREIRA; RODRIGUES, 2016). Dentro dos objetivos do SUS são preconizadas ações de promoção da saúde dos assistidos, instruindo a população a modificarem seus determinantes de saúde em prol da própria qualidade de vida (BRASIL, 1990). Desta forma, seus objetivos e princípios são aplicados com meIhor eficácia à prática assistencial pública mediante a educação em saúde (SANTOS; ANDRADE; LIMA et al., 2012). Um exemplo dessas práticas na área da Nutrição é a educação alimentar e nutricional.

A educação alimentar e nutricional tem por objetivo educar de maneira a abranger o contexto familiar e social, contribuir para escolhas conscientes sobre uma alimentação saudável, e que o educando compreenda o porquê e como realizá-las dentro de suas possibilidades, criando-se assim algo que passe do imaginável para a realidade. Tais ações podem ser direcionadas a diferentes condições clínicas, como a desnutrição e a obesidade (SANTOS, 2005; RAMOS et al., 2013).

Contudo, o presente relatório tem como objetivo descrever as atividades e ações desenvolvidas pelo projeto de extensão direcionado a crianças e gestantes de alto risco realizadas pela Faculdade de Nutrição da Universidade Federal de Pelotas (UFPel). 
O projeto "Assistência nutricional ambulatorial a crianças" é desenvolvido desde 2011 pela Faculdade de Nutrição, junto ao ambulatório de Pediatria da Faculdade de Medicina (FAMED) da UFPel, e realiza atendimento ambulatorial a crianças e gestantes encaminhados pelos Serviços de Pediatria e Ginecologia da FAMED e de unidades básicas de saúde da cidade de Pelotas e região.

$O$ atendimento é realizado semanalmente mediante agendamento, por equipe formada de acadêmicos da disciplina de Nutrição Materno Infantil, bolsistas (extensão, ensino e iniciação científica) e residentes do Programa de Atenção à Saúde da Criança do Hospital Escola da UFPel, supervisionados por docentes da Faculdade de Nutrição.

\section{Atendimentos ambulatorial a crianças}

No atendimento individual para crianças ocorre com o acompanhamento dos pais ou responsáveis. $O$ roteiro da consulta considera o contexto biopsicossocial da criança, a condição nutricional e fisiopatológica, e constitui-se de: anamnese, avaliação de hábitos alimentares, antropométrica (peso, altura e circunferência da cintura) e do estado nutricional.

$\mathrm{Na}$ avaliação antropométrica, as medidas são aferidas por balança digital pediátrica (Digipeso DP-3000 plus, SP - Brasil), estadiômetro (Chasmors Limited, Londres) ou balança digital acoplada ao estadiômetro (Trentin/SC, Brasil). O diagnóstico antropométrico é avaliado pelas curvas de crescimento e desenvolvimento da Organização Mundial de Saúde (OMS, 2006; OMS, 2007) utilizando-se os programas informáticos ANTHRO ou ANTHRO Plus, para crianças menores e maiores de cinco anos, respectivamente (WHO, 2011). Em pacientes com paralisia cerebral são utilizadas curvas especificas de acordo com o sexo e faixa etária com esta condição (DAY; STRAUSS; VACHON et al., 2007).

Ao final do atendimento, as orientações alimentares são conduzidas por definição de metas para a criança, pais/responsáveis e família. O diagnóstico nutricional antropométrico é discutido com os responsáveis, sendo fornecido um laudo para o acompanhamento da evolução dos índices e indicadores. Os retornos às consultas são agendados com intervalos de 15 ou no máximo 30 dias, dependendo do motivo da consulta, metas e proposta de orientações prescritas.

Além de crianças com diagnóstico de obesidade e desnutrição, também são recebidos encaminhamentos de crianças com diagnóstico de autismo, transtorno de déficit de atenção com hiperativi- 
dade, neuropatias, déficit motor associado à prematuridade, além de encaminhamentos específicos para o manejo da amamentação e a orientação da introdução de alimentos no primeiro ano de vida.

$\mathrm{O}$ cuidado com as condutas e a qualidade do serviço ocorre mediante reuniões periódicas entre a equipe. Os protocolos de assistência nutricional são sistematicamente revisados e alterados sempre que necessário visando qualificar a assistência e melhorar a adesão dos usuários as orientações/recomendações nutricionais. No ano de 2016 foram revisadas e ampliadas as anamneses destinadas a pacientes com paralisia cerebral, autistas ou com síndrome de Down. Ainda, foram elaboradas orientações nutricionais direcionadas a esta população especifica de pacientes.

\section{Atendimento multidisciplinar a crianças}

As ações multidisciplinares constituem-se de atividades lúdicas e atendimento individual com os pais/responsáveis. Tais atividades são realizadas em dias pré-determinados, direcionadas a crianças e adolescentes encaminhados por sobrepeso e/ou obesidade, de acordo com a faixa etária. Nestes dias, crianças/adolescentes são direcionadas a um espaço mais amplo da instituição (auditório), onde desenvolvem atividades lúdicas e educacionais relacionadas à alimentação, como: apresentação dos grupos de alimentos, colagem de figuras de alimentos que consideram adequados ou não a saudáveis; reconhecimento dos alimentos através do tato; apresentação de vídeos educativos; pinturas ou elaboração de desenhos seguida de discussões dos alimentos e suas preferências; aula sobre produção e consumo de alimentos in natura.

Paralelamente, os pais/responsáveis são encaminhados para realizarem a anamnese da criança, podendo-se abordar de forma mais profunda o contexto familiar, a rotina da criança, dificuldades enfrentadas na educação, alimentação, cuidado com o filho e família. Ao final, trabalha-se quanto às complicações da obesidade, não somente da criança, como também na família; como e porque criar hábitos saudáveis; a responsabilidade dos pais/responsáveis com a educação nutricional, a importância da atividade física regular com estímulo a brincadeiras mais ativas; também é motivado o retorno para o acompanhamento nutricional, preferencialmente neste novo modelo apresentado. 


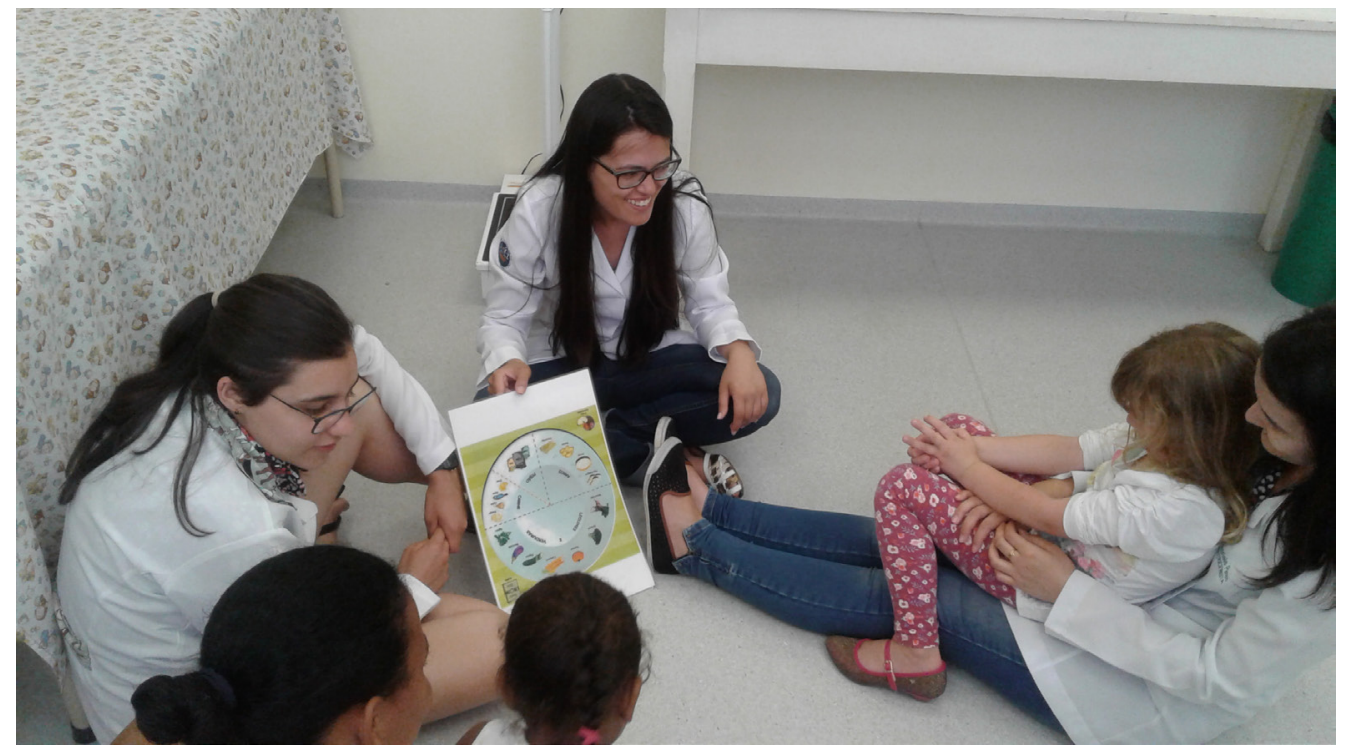

IMAGEM 1

Atividade multidisciplinar realizada pelo projeto "Assistência nutricional ambulatorial de crianças". Faculdade de Nutrição, UFPel, 2016.

Fonte: Arquivo do coordenador do projeto.

\section{Ações em saúde bucal}

Nos encontros multidisciplinares são realizadas atividades referentes à saúde bucal com o uso de vídeos educativos com conteúdo de promoção de saúde, fornecimento de kits de higiene bucal para os participantes e escovação supervisionada. Em atendimentos individualizados, o residente da área da odontologia atende as solicitações de avaliação e orientação de cuidado e higiene bucal de pacientes do serviço de Nutrição e do serviço de Pediatria. A atividade realizada pela odontologia tem importância nutricional, pois nesta faixa etária é relevante prevenir as cáries, melhorar a mastigação e investigar possíveis problemas como a frenectomia (língua presa), os quais interferem na alimentação.

\section{Ações na assistência nutricional pré-natal}

As supervisões para o pré-natal são realizadas com consultas individuais constituída por anamnese nutricional específica para gestante. São avaliados a história obstétrica, o consumo e as preferências alimentares, e a ocorrência de alterações gastrointestinais (sobretudo náuseas, vômitos e constipação). A avaliação antropométrica (peso e altura) e do 
estado nutricional pré-gestacional e atual, e a adequação no ganho de peso são avaliados segundo as recomendações do Institute of Medicine (IOM, 2009).

As orientações nutricionais são prescritas a partir da avaliação e necessidade de adequação dietética. As metas são definidas de acordo com a necessidade de adequação dos hábitos alimentares, estado nutricional, idade gestacional e a previsão de retorno.

O primeiro agendamento ocorre no máximo em quinze dias do encaminhamento, e os retornos são marcados em intervalos de quinze semanas a dois meses, de acordo com a semana gestacional e a presença de complicações como o diabetes gestacional, desordens hipertensivas e o ganho de peso excessivo.

\section{Análises estatísticas}

O serviço de Nutrição realiza o levantamento anual das informações referente aos agendamentos e o estado nutricional de todos os pacientes assistidos, incluindo-se aqui também os atendimentos para a saúde bucal realizados pela dentista residente vinculada ao projeto. No ano de 2016, a partir do total de agendamentos foram estimadas as frequências dos agendamentos, ausências, motivo dos encaminhamentos, faixa etária e, para gestantes, a idade gestacional (trimestre) do encaminhamento.

Todos os dados foram digitados em planilhas Excel (Microsoft Excel, versão 14.0.0.) e, posteriormente, exportados para o pacote estatístico STATA (Stata Corp, College Station, Texas, USA, versão 12.0). Foram conduzidas análises de frequência e gráficos de adequação do estado nutricional de crianças e gestantes.

Para a avaliação do estado nutricional das crianças, os dados referentes aos índices de peso para idade, estatura para idade e índice de massa corporal (IMC) para idade foram extraídos dos programas informáticos ANTRO e ANTRO plus da OMS e as frequências de adequação foram calculadas para cada um dos referidos índices, estratificando-se a população infantil pela faixa etária de menores e maiores de cinco anos de idade. O estado nutricional de gestantes foi avaliado de acordo com os pontos de corte do IMC pré-gestacional. O cálculo deste índice foi realizado a partir da informação do peso pré-gestacional $(\mathrm{em} \mathrm{Kg}$ ) divido pela altura (em metros) ao quadrado, e categorizado em baixo peso $(<18,5 \mathrm{~kg} / \mathrm{m} 2)$, adequado (18,5 a 24,9 kg/m2), sobrepeso (25 a 29,9 kg/m2) ou obesidade (>30 kg/m2). 
No ano de 2016, o serviço de Nutrição recebeu 191 agendamentos de crianças, sendo $56 \%$ consultas novas e $44 \%$ retornos. A maior parte $(65 \%)$ dos encaminhamentos médicos foi justificada pelo diagnóstico de sobrepeso ou obesidade com comorbidades crônicas (dislipidemias, alterações na glicemia ou pressão arterial), seguido de casos de autismo, transtorno do déficit de atenção com hiperatividade (TDHA) ou neuropatias (13\%), erros alimentares ( $8 \%)$, desnutrição (6\%), entre outros.

Do total de crianças que compareceu as consultas $(n=93), 75 \%$ das presenças registradas foram de pacientes de retorno. As faixas etárias atendidas foram lactentes (zero a 2 anos) (6\%), pré-escolares (2 a 5 anos) (34\%), escolares (6 a 10 anos) (30\%) e adolescentes (11 a 19 anos) $(30 \%)$.

Nos Gráficos 1 e 2 estão apresentados a classificação do estado nutricional das crianças. Em relação às crianças menores de cinco anos, os resultados mostram que $33 \%$ das crianças apresentaram peso elevado para a idade, e o índice de IMC para a idade identificou $28 \%$ com sobrepeso e $22 \%$ com obesidade. No que se refere a adequação da estatura, $83 \%$ das crianças foram classificadas com estatura adequada para a idade (Gráfico 1).

\section{Gráfico 1}

Avaliação nutricional das crianças menores de 5 anos a partir de parâmetros antropométricos. Ambulatório de Nutrição Materno Infantil, Universidade Federal de Pelotas, Pelotas, 2016. A) Peso para a Idade. B) Estatura para Idade. C) Peso para Estatura. D) IMC para Idade.

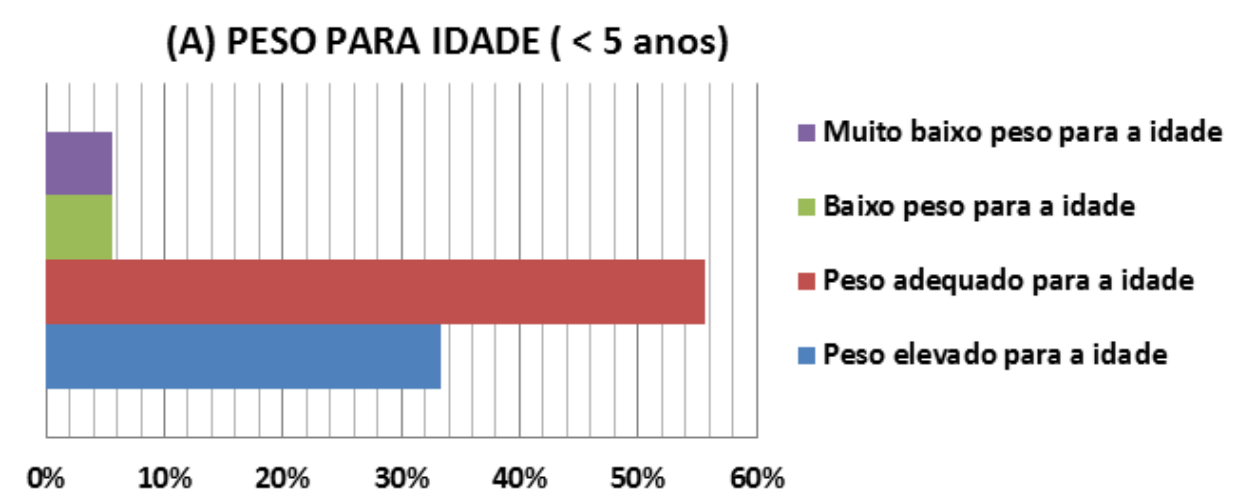


(B) ESTATURA PARA IDADE ( $<5$ anos)

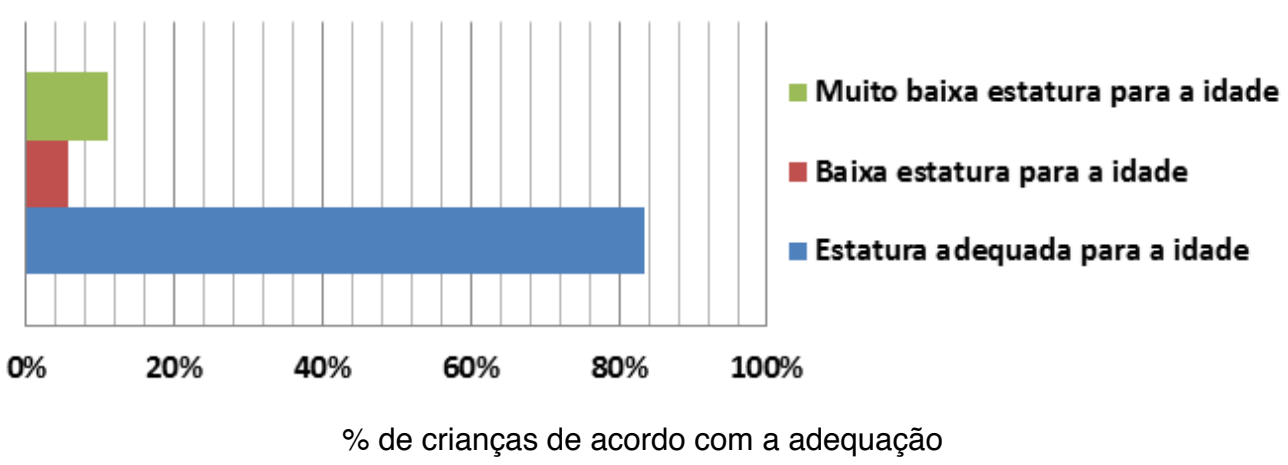

(C) PESO PARA ESTATURA ( $<5$ anos)

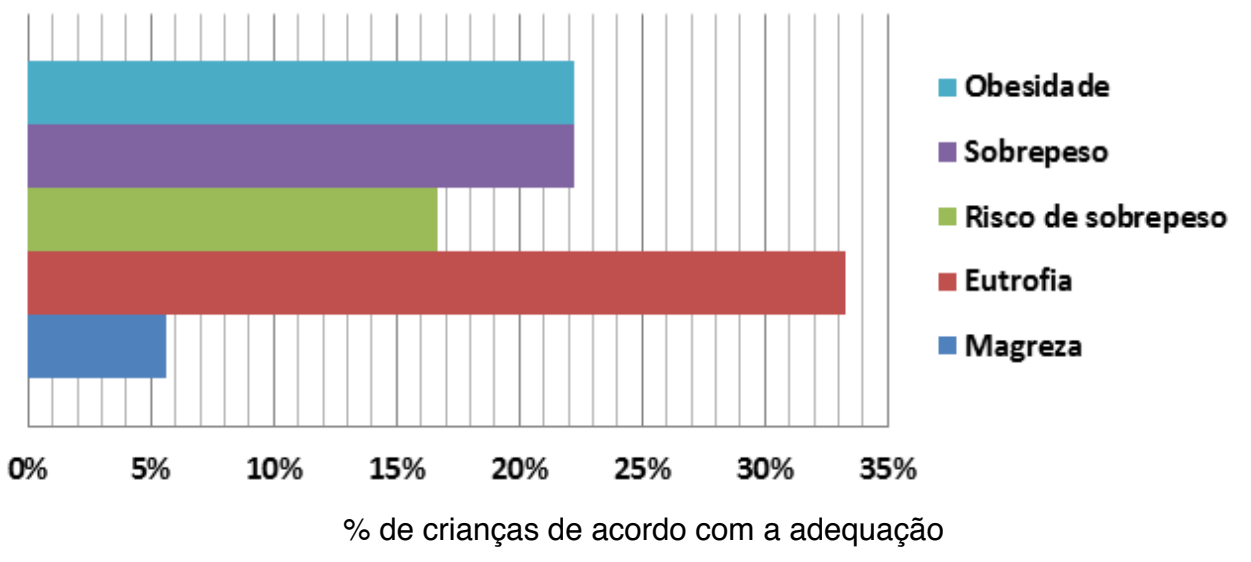

(D) IMC PARA IDADE ( $<5$ anos)

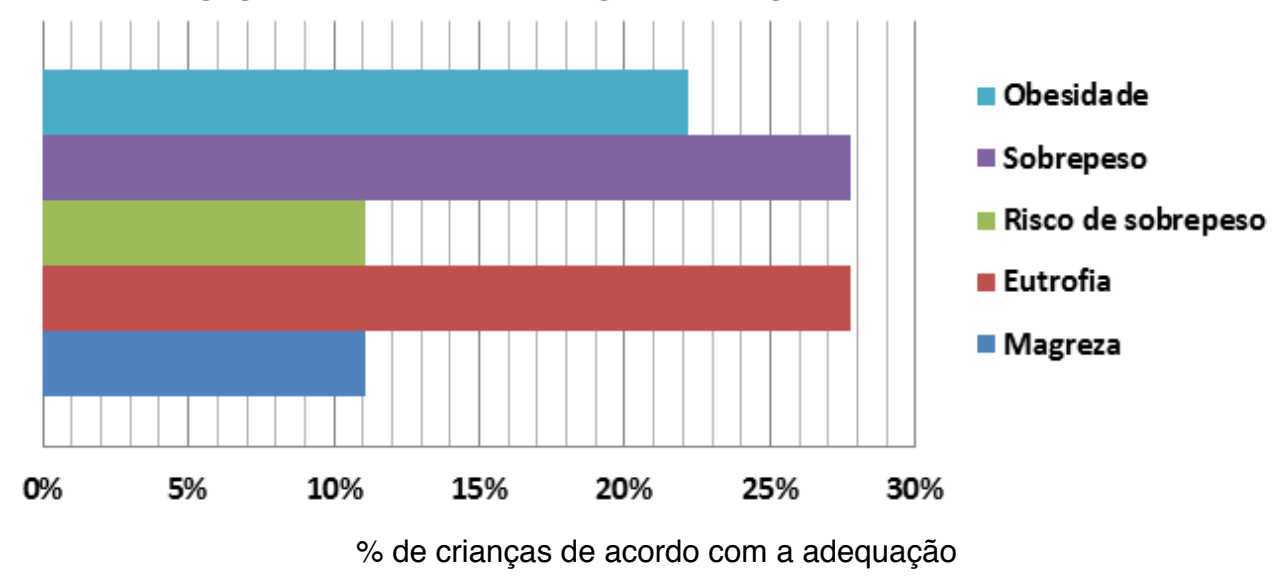

Para as crianças maiores de cinco anos, $45 \%$ foram classificadas com peso elevado para a idade, sendo que no índice de IMC para idade a maioria foi classificada com obesidade $(45 \%)$, obesidade grave $(29 \%)$ ou sobrepeso (15\%). A maioria (94\%) das crianças apresentou a estatura adequada para a idade (Gráfico 2). 


\section{Gráfico 2}

Avaliação nutricional das crianças maiores de 5 anos a partir de parâmetros antropométricos. Ambulatório de Nutrição Materno Infantil, Universidade Federal de Pelotas, Pelotas, 2016. A) Peso para a Idade. B) Estatura para Idade. C) IMC para Idade.

\section{(A) PESO PARA IDADE ( $>5$ anos)}

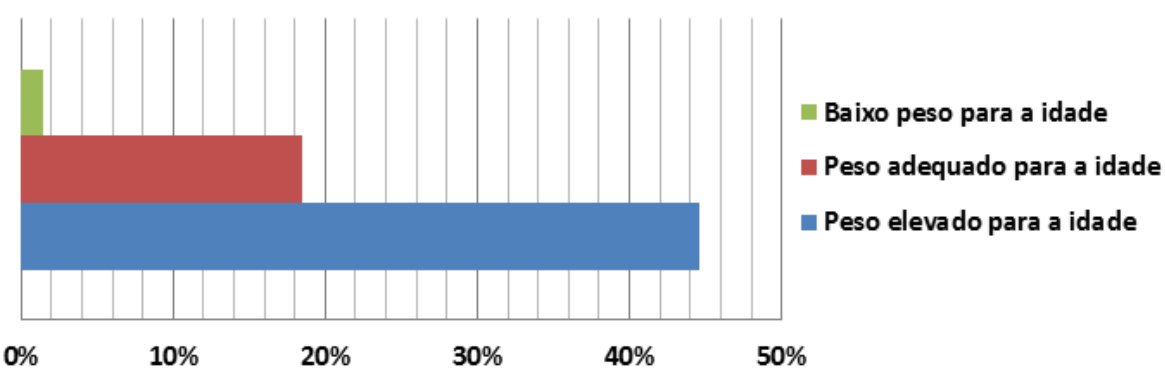

\% de crianças de acordo com a adequação

(B) ESTATURA PARA IDADE ( $>5$ anos)

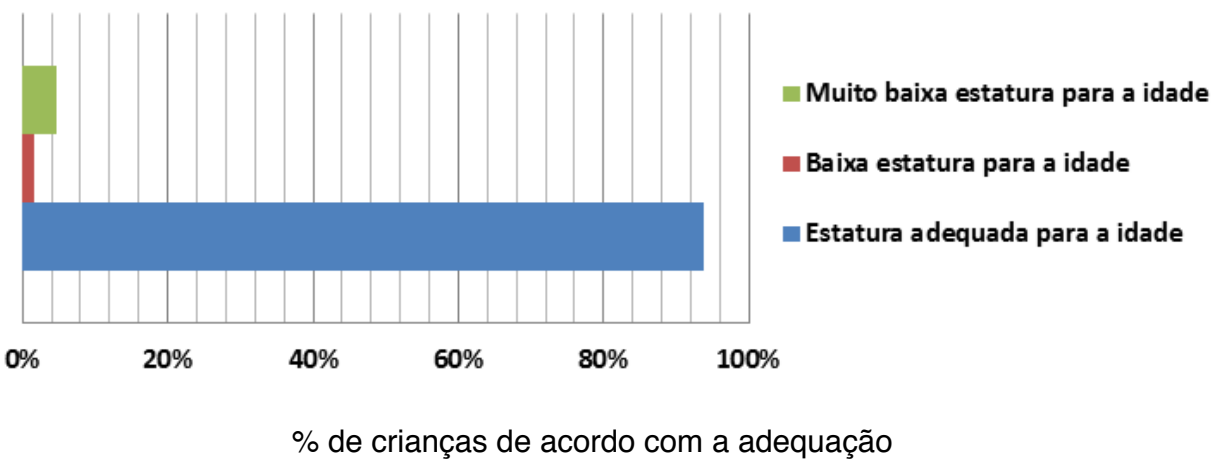

(C) IMC PARA IDADE ( > 5 anos)

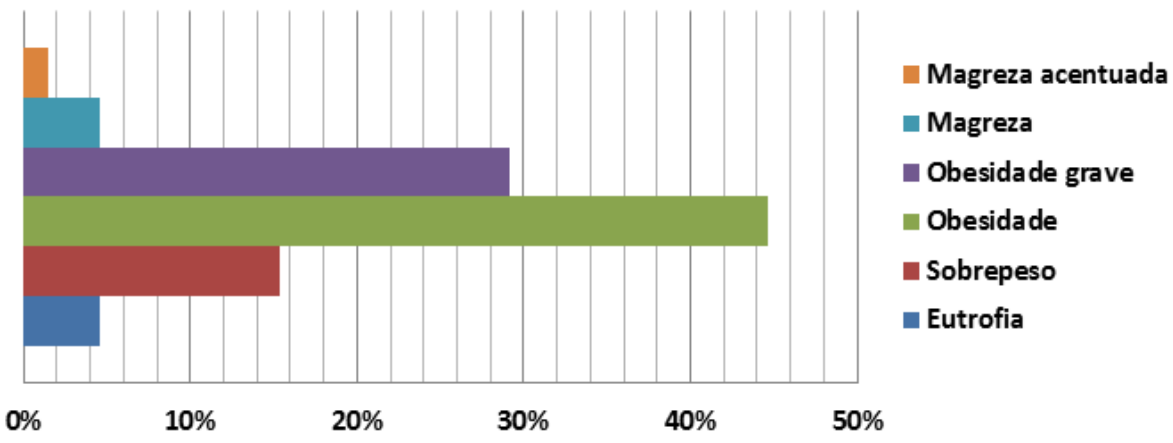

\% de crianças de acordo com a adequação 
Em relação ao atendimento nutricional a gestantes, foram realizados 49 agendamentos no período. Quarenta e quatro por cento das gestantes atendidas eram adultas de 20 a 30 anos e 17\% eram adolescentes. Quanto à idade gestacional, $29 \%$ das pacientes estavam no primeiro trimestre $\mathrm{e}$ $43 \%$ no segundo trimestre. A maioria $(67 \%)$ das gestantes foi encaminhada pelo diagnóstico de sobrepeso ou obesidade. $\mathrm{Na}$ avaliação nutricional, $46 \%$ das gestantes foram classificadas com obesidade pré-gestacional, encaminhadas para a adequação do ganho de peso gestacional. A avaliação do estado nutricional das gestantes está apresentada no Gráfico 3.

\section{Gráfico 3}

Estado nutricional pré-gestacional avaliado na primeira consulta de Nutrição no prénatal. Ambulatório de Nutrição Materno Infantil, Universidade Federal de Pelotas, $\mathrm{Pe}$ lotas, 2016.

\section{ESTADO NUTRICIONAL PRÉ-GESTACIONAL}

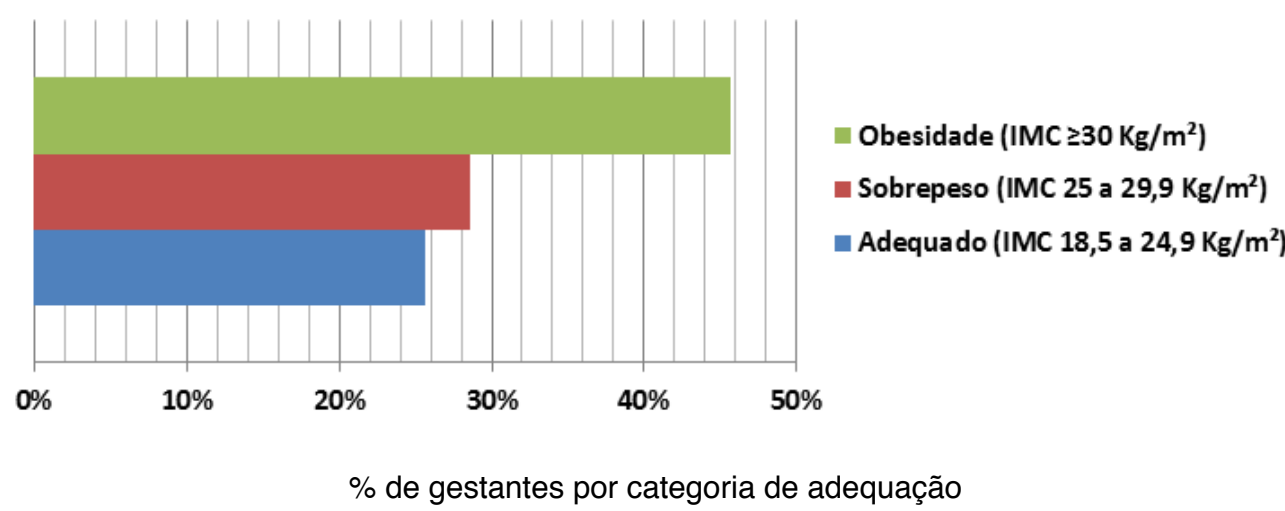

Em relação aos atendimentos para a saúde bucal, 48 encaminhamentos foram registrados a pedido da equipe médica, com $30 \%$ de ausência nas consultas. Outros 40 atendimentos foram realizados juntamente com os atendimentos da Nutrição. Sendo assim, 74 consultas foram efetivamente realizadas em 2016. Do total de atendimentos realizados, $56 \%$ das crianças foram encaminhadas para atendimento odontológico em consultório devido à cárie dentária.

\section{DISCUSSÃO}

O público infantil assistido pelo projeto de extensão do ambulatório de Nutrição apresenta desvios importantes do estado nutricional, visto que a maioria das crianças é encaminhada por sobrepeso ou obesidade na presença de comorbidades como dislipidemias, alterações na glicemia e pressão arterial. A avaliação antropométrica detalhada identifica a 
gravidade do estado nutricional, visto que há um percentual elevado de crianças com obesidade grave. Observa-se ainda que a frequência de obesidade é maior nas crianças acima de cinco anos. Por outro lado, há encaminhamentos por condição de desnutrição que é identificada pelos déficits de peso e estatura. $O$ déficit de peso para a estatura indica uma desnutrição aguda em que a carência nutricional é recente. Já o índice de estatura para a idade revela uma condição de desnutrição crônica, ou seja, indica deficiências cumulativas de saúde e nutrição durante um longo período (WHO, 2006). Apesar da baixa frequência de desnutrição, crianças nesta condição requerem supervisão e intervenção nutricional intensificada.

Estudos conduzidos com população infantil assistidas em creches, escolas e ambulatórios públicos revelam estatísticas semelhantes de obesidade e desnutrição crônica (TRONCON et al, 2007; SILVA et al, 2014). Em um estudo conduzido por Troncon et al. (2007) com a avaliação do estado nutricional de crianças atendidas em um ambulatório universitário, os autores observaram que o diagnóstico de sobrepeso e obesidade era independente dosexo, classificaçãosocioeconômicaeníveldeatividadefísica das crianças.

Quanto à saúde bucal, a maioria das crianças necessitou de encaminhamento para atendimento odontológico em consultório. Sabe-se que a alimentação e nutrição podem influenciar na determinação do processo saúde-doença bucal. Um estudo que acompanhou crianças desde o nascimento na cidade de Porto Alegre observou que a oferta de doces precoce na alimentação infantil, isto é, entre os seis e doze meses de idade, foi significativamente associado à maior incidência de cáries aos seis anos de idade (CHAFFEE; FELDENS; RODRIGUES et al, 2015). Outro estudo realizado com crianças de baixa renda avaliou a relação do custo com a alimentação oferecida e a ocorrência de cáries. Os autores observaram que a cárie estava relacionada com elevado gasto na aquisição de chocolates, refrigerantes e snacks para a alimentação das crianças (FELDENS; RODRIGUES; RAUBER et al., 2013). Tais achados na literatura reforçam que o cuidado na saúde oral infantil necessita de uma atuação multiprofissional, ou seja, todos os profissionais de saúde que atendem o público infantil devem desenvolver ações para de saúde bucal e prevenção da cárie dentária, incluindo a orientação aos pais e responsáveis.

Em relação ao estado nutricional das gestantes assistidas, observouse que a maioria apresentava sobrepeso ou obesidade anterior a gestação. Dados nacionais indicam que a elevada prevalência de excesso de peso em mulheres que ingressam no pré-natal de baixo risco é reflexo da transição nutricional na população brasileira, isto é, aumento na prevalência de obesidade e redução da desnutrição (NIQUINI; BITTENCOURT; LACERDA et al., 2012). Sabe-se que a obesidade materna pré-gestacional é um fator de risco para complicações maternas, aumento na adiposidade fetal e complicações no parto (KUBO; FERRARA; WINDHAM et al., 2014). 
Apesar dos problemas nutricionais estarem contemplados nos manuais de assistência pré-natal do Ministério da Saúde, como o Manual do Pré-Natal e Puerpério (Ministério da Saúde, 2006) e do Caderno de Atenção Básica do Programa de Pré-Natal de Baixo Risco (BRASIL. Ministério da Saúde, 2012), ainda não há uma orientação estabelecida do encaminhamento das gestantes para o atendimento de Nutrição (NIQUINI; BITTENCOURT; LACERDA et al., 2012), mesmo em serviços de atenção a gestantes de alto risco. A supervisão nutricional durante o pré-natal possibilita planejar de forma adequada o ganho de peso ao longo da gestação de acordo com o estado nutricional pré-gestacional, sendo possível manejar o ganho de peso de acordo com as recomendações mesmo em situações de obesidade anterior a gestação (IOM, 2009).

\section{CONCLUSÃO}

As ações deste projeto preenchem uma lacuna importante na assistência ambulatorial de crianças e gestantes de alto risco na comunidade de Pelotas e região. $\mathrm{O}$ atendimento ambulatorial como extensão universitária promove a interação entre a universidade e a comunidade, prestando serviços, nos quais ambas as partes são beneficiadas. Para os estudantes, o projeto é uma oportunidade de vivenciar a prática; para a comunidade, uma oportunidade de recebe um atendimento em constante aperfeiçoamento. Desta forma realiza-se parte da missão das universidades em promover melhoria da qualidade de vida. A prática proporciona uma gama de situações onde o estudante é estimulado e encorajado com novos conhecimentos e desafios que complementam o ensino em sala de aula.

\section{REFERÊNCIAS}

BLÖSSNER, M.; SIYAM, A.; BORGHI, E. et al. Software for assessing growth and development of the world's children. World Health Organization. Department of Nutrition for Health and Development. Switzerland, 2011. Disponível em: <http://www.who.int/childgrowth/software/en/>. Acesso em: 7 dez. 2016.

BRASIL. Lei n 8080 de 19 de setembro de 1990. Dispõe sobre as condições para a promoção, proteção e recuperação da saúde, a organização e o funcionamento dos serviços correspondentes e dá outras providências.

Diário Oficial da República Federativa do Brasil, set.1990. 
BRASIL. Ministério da Saúde. Pesquisa Nacional de Demografia e Saúde da Criança e da Mulher - PNDS 2006: dimensões do processo reprodutivo e da saúde da criança. Brasília; 2009.

BRASIL. Ministério da Saúde. Secretaria de Atenção à Saúde. Departamento de Ações Programáticas Estratégicas. Pré-natal e puerpério: atenção qualificada e humanizada: manual técnico. Brasília, 2006. Disponível em: <http://portal.saude.gov.br/portal/arquivos/pdf/Manual\%20Puerperio\%202006>. Acesso em: 16 jul. 2016.

BRASIL. Ministério da Saúde. Secretaria de Atenção à Saúde. Departamento de Atenção Básica. Cadernos de atenção básica: atenção ao prénatal de baixo risco. n. 32. Brasília: 2012.

BRASIL. Relatório da Comissão pelo Fim da Obesidade Infantil busca reverter aumento de sobrepeso e obesidade. Organização Mundial da Saúde, Brasília, 2016. Disponível em: <http://www.paho.org/bra/index.php? option=com_content\&view=article\&id=4997:relatorioda-comissao-pelo-fim -da-obesidade-infantil-busca-reverter-aumentode-sobrepeso-eobesidade \&ltemid=821 $>$. Acesso em: 15 jul. 2016.

CABRAL, A.; BRAGAGLIA, A. P.; SEABRA, I. A publicidade infantil no Brasil e suas implicações ético-legais: estudo empírico em campanhas voltadas ao Dia da Criança. Revista Temática, v. 8., n. 12, 2012.

CARVALHO, S. S.; COELHO, J. M. F.; SOARES, D. A. B. et al. Fatores maternos para o nascimento de recém-nascidos com baixo peso e prematuros: estudo caso-controle. Revista Ciência e Saúde, v.9, n.2, p.76-82, 2016.

CHAFFEE, B. W.; FELDENS C. A.; RODRIGUES, P. H. et al. Feeding practices in infancy associated with caries incidence in early childhood. Community dentistry and oral epidemiology, v. 43 , n. 4 , p. $338-348$, 2015.

DAY, S. M.; STRAUSS, D. J.; VACHON, P. J et al. Growth patterns in a population of children and adolescents with cerebral palsy. Developmental Medicine \& Child Neurology, v. 49, p. 167-171, 2007.

DE ONIS, M. et al. Development of a WHO growth reference for school-aged children and adolescents. Bulletin of the World Health Organization, v. 85, p. 660-667, 2007. 
DA SILVA, M. C.; CAPANEMA, F. D.; LAMOUNIER, J. A. et al. Perfil nutricional de crianças pré-escolares em creches públicas de Belo HorizonteMinas Gerais beneficiárias ou não do Programa Bolsa Família. Percurso Acadêmico, v. 4, n. 7, p. 88-104, 2015.

DE SENA, J. N; PEREIRA, M. S; RODRIGUES, R. B. Reflexões sobre saúde, nutrição e estratégia saúde da família no contexto de integralidade do sistema único de saúde (SUS). Revista Rede de Cuidados em Saúde, v. 9, n. 3, p. 1-25, 2016.

DREHMER, M.; DUNCAN, B. B.; KAC, G. et al. Association of second and third trimester weight gain in pregnancy with maternal and fetal outcomes. PLOS One, v. 8, n. 1,: e54704, 2013.

FELDENS, C. A.; RODRIGUES, P. H., RAUBER, F. et al. Food expenditures, cariogenic dietary practices and childhood dental caries in southern Brazil. Caries Research, v. 47, n. 5, p. 373-381, 2013.

IOM. Weight gain during pregnancy: reexamining the guidelines. Institute of Medicine, Washington, 2009. Disponível em: <http://www.nationalacademies.org/hmd/ /media/Files/Report\%20Files/2009/Weight-Gain-During -Pregnancy-Reexamining-the-Guidelines/Report\%20Brief\%20-\%>. Acesso em: 15 jul. 2016.

KAUFMANN, C. C.; ALBERNAZ, E. P. Prevalência e fatores associados ao excesso de peso em crianças de uma coorte no sul do Brasil. Ciência \& Saúde, v. 6, n. 3, p. 172-180, 2013.

KUBO, A.; FERRARA, A.; WINDHAM, G. C. et al. Maternal hyperglycemia during pregnancy predicts adiposity of the offspring. Diabetes Care, v. 37, n. 11, p. 2996-3002, 2014.

NIQUINI, R. P.; BITTENCOURT, S. A.; LACERDA, E. et al. Avaliação do processo da assistência nutricional no pré-natal em sete unidades de saúde da família do Município do Rio de Janeiro. Ciência e Saúde Coletiva, v. 17, p. $2805-2816,2012$.

RAMOS, F. P.; SANTOS, L. A. S.; REIS, A. B. C. Educação alimentar e nutricional em escolares: uma revisão de literatura. Caderno de Saúde Pública, v. 29, n. 11, p. 2147-2161, 2013.

SALVADOR, C. C. Z.; KITOKO, P. M.; GAMBARDELLA, A. M. D. Nutritional status of children and adolescents: factors associated to overweight 
and fat accumulation. Journal of Human Growth and Development, v. 24, n. 3, p. 313-319, 2014.

SANTOS, D. S.; ANDRADE, A. L. A.; LIMA, B. S. S. et al. Sala de espera para gestantes: uma estratégia de educação em saúde. Revista Brasileira de Educação Médica, v. 36, n. 1, p. 62-67, 2012.

SANTOS, L. A. S. Educação alimentar e nutricional no contexto da promoção de práticas alimentares saudáveis. Revista de Nutrição, v. 18, n. 5, p. 681-692, 2005.

SILVEIRA, F. J. F.; BARBOSA, J. C.; VIEIRA, V. A. M. Prevalência de sobrepeso e obesidade em crianças de quatro escolas estaduais de Belo Horizonte, Minas Gerais. Revista Médica de Minas Gerais, v. 25, n. 2, p. 180-186, 2015.

SILVEIRA, M. F.; SANTOS, I. S.; BARROS, A. J. D. et al. Aumento da prematuridade no Brasil: revisão de estudos de base populacional. Revista de Saúde Pública, v. 42, n. 5, p. 957-964, 2008.

TRONCON, J. K.; GOMES, J. P.; JÚNIOR, G. G. et al. Prevalência de obesidade em crianças de uma escola pública e de um ambulatório geral de pediatria de hospital universitário. Revista Paulista de Pediatria, v. 25, n. 4, p. 305-310, 2007.

VICTORA, C. G. et al. Saúde de mães e crianças no Brasil: progressos e desafios. The Lancet, v. 337, n. 9780, p. 1863-1876, 2011.

WORLD HEALTH ORGANIZATION. Child growth standards: length/height-for-age, weight-for-age, weight-for-length, weight-for-height and body mass index-for-age. Methods and development. WHO (non serial publication). Geneva: WHO, 2006.

Data de recebimento: 30 de março de 2017.

Data de aceite para publicação: 15 de maio de 2017. 